\title{
Glucose Outcomes with the In-Home Use of a Hybrid Closed-Loop Insulin Delivery System in Adolescents and Adults with Type 1 Diabetes
}

\author{
Satish K. Garg, MD, ${ }^{1, *}$ Stuart A. Weinzimer, $M D^{2}$ William V. Tamborlane, $M D^{2}$ \\ Bruce A. Buckingham, MD, Bruce W. Bode, MD, Timothy S. Bailey, MD, Ronald L. Brazg, MD, \\ Jacob Ilany, MD, Robert H. Slover, MD, Stacey M. Anderson, MD, Richard M. Bergenstal, MD, \\ Benyamin Grosman, $\mathrm{PhD}^{10}$ Anirban Roy, $\mathrm{PhD}^{10}$ Toni L. Cordero, $\mathrm{PhD},{ }^{10}$ \\ John Shin, PhD, MBA, ${ }^{10}$ Scott W. Lee, MD, ${ }^{10}$ and Francine R. Kaufman, MD ${ }^{10}$
}

\begin{abstract}
Background: The safety and effectiveness of the in-home use of a hybrid closed-loop (HCL) system that automatically increases, decreases, and suspends insulin delivery in response to continuous glucose monitoring were investigated.

Methods: Adolescents ( $n=30$, ages 14-21 years) and adults ( $n=94$, ages 22-75 years) with type 1 diabetes participated in a multicenter (nine sites in the United States, one site in Israel) pivotal trial. The Medtronic MiniMed ${ }^{\mathbb{P}} 670 \mathrm{G}$ system was used during a 2-week run-in phase without HCL control, or Auto Mode, enabled (Manual Mode) and, thereafter, with Auto Mode enabled during a 3-month study phase. A supervised hotel stay (6 days/5 nights) that included a 24-h frequent blood sample testing with a reference measurement (i-STAT) occurred during the study phase. Results: Adolescents (mean \pm standard deviation [SD] 16.5 \pm 2.29 years of age and $7.7 \pm 4.15$ years of diabetes) used the system for a median $75.8 \%$ (interquartile range [IQR] 68.0\%-88.4\%) of the time (2977 patient-days). Adults (mean \pm SD $44.6 \pm 12.79$ years of age and $26.4 \pm 12.43$ years of diabetes) used the system for a median $88.0 \%$ (IQR 77.6\%-92.7\%) of the time (9412 patient-days). From baseline run-in to the end of study phase, adolescent and adult $\mathrm{HbA}_{1 \mathrm{c}}$ levels decreased from $7.7 \% \pm 0.8 \%$ to $7.1 \% \pm 0.6 \%(P<0.001)$ and from $7.3 \% \pm$ $0.9 \%$ to $6.8 \% \pm 0.6 \%(P<0.001$, Wilcoxon signed-rank test $)$, respectively. The proportion of overall in-target $(71-180 \mathrm{mg} / \mathrm{dL})$ sensor glucose $(\mathrm{SG})$ values increased from $60.4 \% \pm 10.9 \%$ to $67.2 \% \pm 8.2 \%(P<0.001)$ in adolescents and from $68.8 \% \pm 11.9 \%$ to $73.8 \% \pm 8.4 \%(P<0.001)$ in adults. During the hotel stay, the proportion of in-target i-STAT ${ }^{\circledR}$ blood glucose values was $67.4 \% \pm 27.7 \%$ compared to SG values of $72.0 \% \pm 11.6 \%$ for adolescents and $74.2 \% \pm 17.5 \%$ compared to $76.9 \% \pm 8.3 \%$ for adults. There were no severe hypoglycemic or diabetic ketoacidosis events in either cohort.
\end{abstract}

\footnotetext{
${ }^{1}$ Barbara Davis Center for Diabetes, University of Colorado Denver, Aurora, Colorado.

${ }^{2}$ Yale University, New Haven, Connecticut.

${ }^{3}$ Stanford University, Stanford, California.

${ }_{5}^{4}$ Atlanta Diabetes Associates, Atlanta, Georgia.

${ }^{5}$ AMCR Institute, Escondido, California.

${ }^{6}$ Rainier Clinical Research Center, Renton, Washington.

${ }^{7}$ Sheba Medical Center, Tel Hashomer, Israel.

${ }^{8}$ University of Virginia, Charlottesville, Virginia.

${ }^{9}$ International Diabetes Center, Minneapolis, Minnesota.

${ }^{10}$ Medtronic, Northridge, California.

*Editor-in-Chief, DTT.

(c) Satish K. Garg et al., 2017; Published by Mary Ann Liebert, Inc. This Open Access article is distributed under the terms of the Creative Commons Attribution Noncommercial License (http://creativecommons.org/licenses/by-nc/4.0/) which permits any noncommercial use, distribution, and reproduction in any medium, provided the original author(s) and the source are credited.
} 
Conclusions: HCL therapy was safe during in-home use by adolescents and adults and the study phase demonstrated increased time in target, and reductions in $\mathrm{HbA}_{1 \mathrm{c}}$, hyperglycemia and hypoglycemia, compared to baseline. Trial Registration: Clinicaltrials.gov identifier: NCT02463097.

Keywords: Hybrid closed loop, Insulin pump, Continuous glucose monitoring, Type 1 diabetes, Hyperglycemia, Hypoglycemia, Sensor.

\section{Introduction}

D ESPITE MANY ADVANCES in type 1 diabetes therapies, most patients are unable to achieve near-normal glycemia, ${ }^{1}$ and remain at risk for severe hypoglycemia, diabetic ketoacidosis, and long-term vascular complications. Closed-loop automated insulin delivery technology uses a control algorithm to automatically increase, decrease, and suspend insulin delivery using subcutaneous glucose sensor data, to improve glucose control and lessen the burden of diabetes management. Due to the absorption profile of rapid-acting insulin analogues, most closed-loop systems under development are hybrid systems, meaning that the user is still required to estimate meal carbohydrate content and confirm the recommended bolus insulin dose calculated from the carbohydrate-to-insulin ratio, before initiating bolus insulin delivery. ${ }^{2}$

Hybrid closed-loop (HCL) systems use various combinations of control algorithms, glucose sensors, and insulin pumps. ${ }^{3}$ Early studies of automated insulin delivery systems, many of which were overnight devices, were conducted in silico $^{4}$ or under controlled inpatient conditions. ${ }^{5-10}$ Later studies, in supervised outpatient settings such as hotels ${ }^{11}$ and camps with subjects as young as 10 years, ${ }^{12,13}$ showed that automated insulin delivery systems increased the time in which sensor glucose (SG) values were in the target range, variously defined as $>70$ to 145 or $180 \mathrm{mg} / \mathrm{dL}$. Closed-loop systems have also been evaluated in the unmonitored inhome setting in a limited number of studies. ${ }^{14-18}$ Medtronic systems have been under development for several years. ${ }^{19}$ Feasibility or preliminary studies in clinical settings, ${ }^{20}$ at camp, $^{21}$ in hotel, ${ }^{22}$ and in supervised outpatient settings ${ }^{23}$ have been carried out to evaluate the robustness of the Medtronic control algorithm, including the system's response to unannounced meals and exercise, and disrupted sensor/ pump communications. The safety of constraining the maximal insulin delivery rate that the system can give automatically has also been assessed. ${ }^{24}$ As a result of the positive outcomes in these feasibility studies, the HCL system was studied in a pivotal trial of sufficient size (124 adolescents and adults) and duration (3 months) to demonstrate its safety. While a brief summary of safety endpoints has been previously reported, ${ }^{25}$ a more complete description of the study's secondary and exploratory outcomes is presented herein.

\section{Materials and Methods}

The study was conducted at 10 sites ( 9 in the United States and 1 in Israel) and enrolled 129 subjects (32 adolescents 14 21 years, and 97 adults $22-75$ years) with type 1 diabetes for $\geq 2$ years, $\mathrm{HbA}_{1 \mathrm{c}}$ value $<10 \%$, and who had used insulinpump therapy for $>6$ months with or without continuous glucose monitoring (CGM) experience. The primary endpoint of the study was safety of HCL system use. All inclusion and exclusion criteria are listed in the Supplementary
Data (Supplementary Data are available online at www .liebertpub.com/dia). The study obtained institutional review board approvals and written informed consent from adults and the parents or guardians of minors. Written assent was obtained from all minors.

Enrolled subjects were trained on the HCL system (Medtronic, Northridge, CA) that included the Guardian ${ }^{\circledR}$ Sensor 3 glucose sensor with Guardian Link 3 transmitter, the MiniMed 670G insulin pump with HCL algorithm, and the CONTOUR ${ }^{\circledR}$ NEXT LINK blood glucose meter (Ascensia Diabetes Care, Parsippany, NJ). Subjects were instructed to upload system data via CareLink ${ }^{\circledR}$ Clinical software (Medtronic) every day during the first 2 weeks that Auto Mode was enabled, then weekly thereafter. The data were reviewed by the research team to verify that subjects were using the system appropriately (i.e., enabling Auto Mode, applying setting adjustments, and changing consumables). Site personnel set active insulin time, carbohydrate-to-insulin ratio(s), basal rates, glucose targets, and sensitivity factors for Manual Mode. Active insulin time and carbohydrate-to-insulin ratio(s) were carried over when Auto Mode was enabled. Automatic changes to the variable basal rate delivered by microboluses, as well as correction doses, were adjusted by the HCL system algorithm. During HCL control, insulin boluses could only be delivered by a carbohydrate announcement or by a finger stick blood glucose value if doing a correction bolus.

The recommended initial high alert setting was $300 \mathrm{mg} /$ $\mathrm{dL}$ and low alert setting was $70 \mathrm{mg} / \mathrm{dL}$ for Manual Mode and Auto Mode control. In addition, a fixed alarm provided alerts when SG values reached $50 \mathrm{mg} / \mathrm{dL}$, after $1 \mathrm{~h}$ of $>300 \mathrm{mg} / \mathrm{dL}$ and after $3 \mathrm{~h}$ of $>250 \mathrm{mg} / \mathrm{dL}$. The algorithm's glucose target was fixed at $120 \mathrm{mg} / \mathrm{dL}$, with an optional temporary target of $150 \mathrm{mg} / \mathrm{dL}$ for exercise that could be set by the user.

There was a 2-week in-home period (run-in phase), in which subjects used the system in Manual Mode followed by a 3-month in-home study phase in which Auto Mode was enabled. Manual Mode data collected during the first 6 days of the study phase provided the basis for initial personalized HCL control parameters. During the study phase, each subject had a 6-day/5-night hotel stay that included $4 \mathrm{~h}$ of exercise each day, and frequent sample testing (FST) of venous blood glucose with a reference instrument (i-STAT ${ }^{\circledR}$ system, Abbott Laboratories, Abbott Park, IL) every 30$60 \mathrm{~min}$ over a $24-\mathrm{h}$ period. The activities during the $4-\mathrm{h}$ exercise session varied at each site and for each subject. The hotel stay was scheduled during each of the 3 months of the study phase. The hotel stay served to verify the accuracy of SG readings with respect to venous blood glucose measurements under near real-world conditions. $\mathrm{HbA}_{1 \mathrm{c}}$ was measured at a National Glycohemoglobin Standardization Program-certified central laboratory. 


\section{HCL system}

The HCL algorithm has been previously described. ${ }^{23,26}$ Subjects were requested to calibrate the sensor a recommended 3-4 times per day, enter carbohydrate estimates for meal boluses, and perform finger stick blood glucose values for correction insulin boluses. The HCL algorithm automatically adjusted multiple parameters based on recent data regarding insulin delivery and glucose levels every midnight. Users could stop HCL control-enabled insulin delivery at any time, or the system could exit from Auto Mode for several reasons that included sensor issues (e.g., a lost sensor signal or a sensor at the end of its functional life), the glucose level (e.g., persistent readings above or below prespecified limits), or insulin delivery (e.g., pump occlusions or persistent delivery rates above or below prespecified limits).

\section{Statistical analyses}

As the primary endpoint for the study was safety, sample size was not determined. All statistical analyses were based on subjects who entered the study phase $(n=124)$. Study phase data were collected throughout the in-home and hotel intervals. Data regarding insulin delivery were consistently available for analysis; data from glucose sensors were occasionally unavailable for reasons including routine sensor initializations and removals. All analyses were considered exploratory and the $P$-values were presented without multiplicity adjustment. Tests were performed with either a Wilcoxon signed-rank test and indicated by a Wilcoxon signed-rank test, or a paired $t$-test, as needed. Statistical analyses were performed using SAS $^{\circledR} 9.4$ (SAS Institute, Cary, NC).

The time period between 10:00 p.m. and 7:00 a.m. was defined as the nighttime interval. Endpoints were averaged per subject and compared between the run-in phase and study phase. These included the percent of SG values in various glucose ranges (i.e., $\leq 50 \mathrm{mg} / \mathrm{dL}, \leq 70 \mathrm{mg} / \mathrm{dL}, 71$ to $180 \mathrm{mg} / \mathrm{dL}$ [in-target range], $>180 \mathrm{mg} / \mathrm{dL}$, and $>300 \mathrm{mg} / \mathrm{dL}$ ); within-day variability of SG values (i.e., within-day standard deviation [SD] and coefficient of variation [CV]); $\mathrm{HbA}_{1 \mathrm{c}}$; insulin delivered (i.e., total daily dose [TDD] and basal insulin [basal and microbolus] as a percent of TDD); body weight; nighttime percent of $\mathrm{SG}$ values in various glucose ranges (i.e., $\leq 50 \mathrm{mg} / \mathrm{dL}, \leq 70 \mathrm{mg} / \mathrm{dL}, 71$ to $180 \mathrm{mg} / \mathrm{dL},>180 \mathrm{mg} / \mathrm{dL}$, and $>300 \mathrm{mg} / \mathrm{dL}$ ); and percent of fasting in-target $(71-180 \mathrm{mg} /$ dL) SG values. Hotel i-STAT blood glucose, SG, and mean absolute relative difference (MARD) were also reported.

\section{Results}

\section{Subjects and system use}

Of the 129 subjects enrolled, there were two screen failures and four withdrawals (a withdrawal rate of less than 5\%). One withdrawal occurred during screening, two during the run-in phase, and one during the study phase. The characteristics of the 124 subjects who entered the study phase are shown in Table 1.

The HCL system was used for 12,389 patient-days (2977 patient-days for adolescents and 9412 patient-days for adults), calculated from the start of system use to the end of the study. Auto Mode was enabled a median $75.8 \%$ (interquartile range [IQR] $68.0 \%-88.4 \%$ ) of the time or $18.2 \mathrm{~h} /$ day
Table 1. Characteristics of the Study Population

\begin{tabular}{lcc}
\hline Characteristic & $\begin{array}{c}\text { Adolescents } \\
(\mathrm{N}=30)\end{array}$ & $\begin{array}{c}\text { Adults } \\
(\mathrm{N}=94)\end{array}$ \\
\hline Female, $N(\%)$ & $16(53.3)$ & $53(56.4)$ \\
Male, $N(\%)$ & $14(46.7)$ & $41(43.6)$ \\
Age, mean $\pm \mathrm{SD}$, years & $16.5 \pm 2.29$ & $44.6 \pm 12.79$ \\
Weight, mean $\pm \mathrm{SD}, \mathrm{kg}$ & $67.4 \pm 12.98$ & $79.9 \pm 18.20$ \\
BMI, mean $\pm \mathrm{SD}, \mathrm{kg} / \mathrm{m}^{2}$ & $23.7 \pm 3.80$ & $27.1 \pm 5.42$ \\
Duration of diabetes, & $7.7 \pm 4.15$ & $26.4 \pm 12.43$ \\
$\quad$ mean $\pm \mathrm{SD}$, years & & \\
TDD, mean $\pm \mathrm{SD}$, & $0.8 \pm 0.24$ & $0.6 \pm 0.20$ \\
$\quad$ U/kg/day & & \\
HbA1C, mean $\pm \mathrm{SD}$, & $7.7 \pm 0.84$ & $7.3 \pm 0.91$ \\
$\quad \%(\mathrm{IQR})$ & $(7.1-8.4)$ & $(6.7-7.8)$ \\
\hline
\end{tabular}

BMI, body mass index; IQR, interquartile range; $\mathrm{SD}$, standard deviation; TDD, total daily dose of insulin.

(IQR 16.3-21.2 h/day) by adolescents; and a median $88.0 \%$ (IQR $77.6 \%-92.7 \%$ ) of the time or $21.1 \mathrm{~h} /$ day (IQR 18.6$22.2 \mathrm{~h}$ /day) by adults. Compared to adolescents, adults demonstrated greater time in Auto Mode. Sensors were used for a median $88.6 \%$ (IQR $81.7 \%-93.4 \%$ ) of the time or $21.3 \mathrm{~h} /$ day (IQR 19.6-22.4 h/day) by adolescents; and a median $93.1 \%$ (IQR $89.2 \%-95.9 \%$ ) of the time or $22.3 \mathrm{~h} /$ day (IQR 21.4 $23.0 \mathrm{~h} /$ day) by adults.

\section{Glucose control, SG variability, $H b A_{1 c}$, insulin delivered, and weight}

SG profiles of adolescents and adults across the day and night, during the run-in and study phases, are shown in Figure 1. The pattern of SG values during the night, for both groups, was similar and revealed less variability during the study phase. The reduced variability was most evident in the adolescent cohort (Fig. 1A). During the day, the HCL system appeared to mitigate hyperglycemia more in the adolescent cohort and hypoglycemia more in the adult cohort (Fig. 1B).

Table 2 shows the run-in phase and study phase mean \pm SD and median (IQR) SG values, percent of SG values in various ranges, and the within-day SD and CV of SG levels. For the adolescent cohort, the percent of mean in-target $\mathrm{SG}$ values increased from $60.4 \% \pm 10.9 \%$ during the run-in phase to $67.2 \% \pm 8.2 \%$ during the study phase $(P<0.001)$, while the values $>180 \mathrm{mg} / \mathrm{dL}$ decreased from $35.3 \% \pm 11.4 \%$ to $30.0 \% \pm 8.0 \%(P<0.001$, Wilcoxon signed-rank test $)$ and $\leq 70 \mathrm{mg} / \mathrm{dL}$ decreased from $4.3 \% \pm 2.9 \%$ to $2.8 \% \pm 1.3 \%$ $(P=0.00928)$ (Table 2). A reduction in mean within-day variability in SG from the run-in phase to the study phase was also observed (SD, $P<0.001$ and $\mathrm{CV}, P=0.00118$ ). For the adult cohort, the percent of in-target $\mathrm{SG}$ values increased from $68.8 \% \pm 11.9 \%$ during the run-in phase to $73.8 \% \pm 8.4 \%$ during the study phase $(P<0.001)$, while the values $>180$ $\mathrm{mg} / \mathrm{dL}$ decreased from $24.9 \% \pm 13.5 \%$ to $22.8 \% \pm 8.9 \%$ $(P=0.01045)$ and $\leq 70 \mathrm{mg} / \mathrm{dL}$ decreased from $6.4 \% \pm 4.3 \%$ to $3.4 \% \pm 2.1 \% \quad(P<0.001$, Wilcoxon signed-rank test $)$ (Table 2). The reduced within-day variability in SG for adults, from the run-in phase to the study phase, was comparable to that observed in adolescents $(P<0.001$, for both SD and CV).

Table 2 also shows that $\mathrm{HbA}_{1 \mathrm{c}}$ levels fell from a mean of $7.7 \% \pm 0.8 \%$ at baseline to $7.1 \% \pm 0.6 \%$ at the end of the 

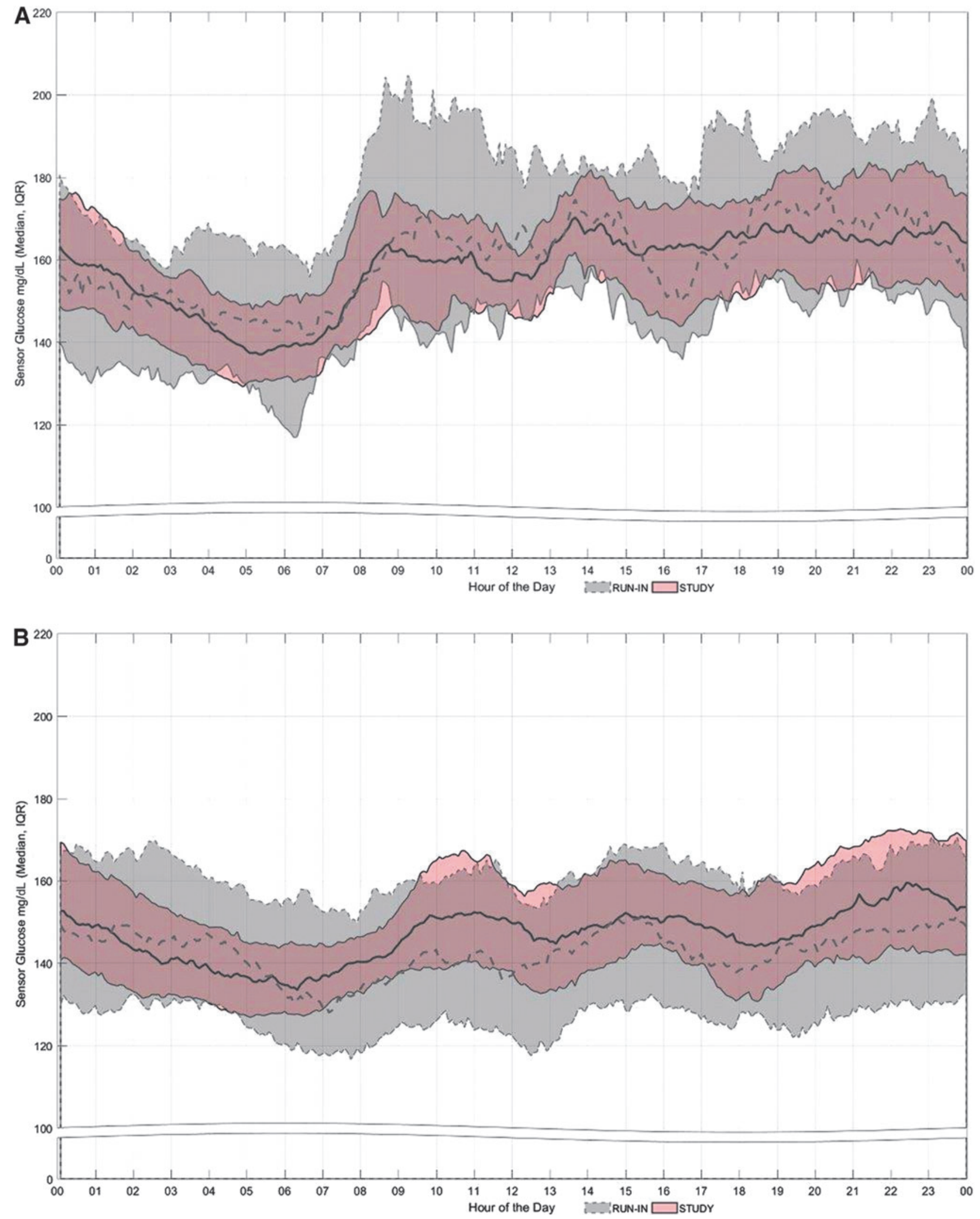

FIG. 1. Sensor glucose profiles during the run-in and study phase. Median and interquartile range of sensor glucose values throughout the day and night, beginning at midnight (00, on $x$-axis), in (A) adolescents and (B) adults. The gray band and dotted line represent data from the run-in phase; the pink band and solid line represent data from the study phase. 


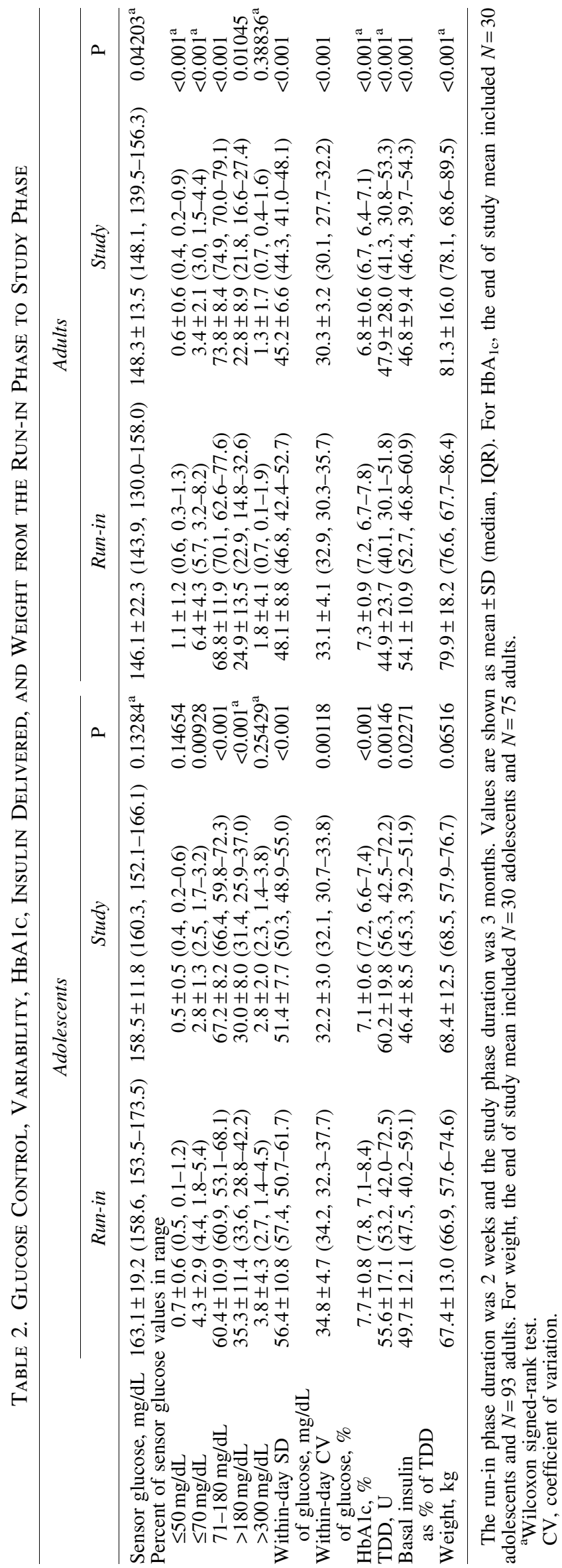

study phase, for adolescents $(P<0.001)$ and from $7.3 \% \pm$ $0.9 \%$ to $6.8 \% \pm 0.6 \%$, for adults $(P<0.001$, Wilcoxon signedrank test). There was a mean change in $\mathrm{HbA}_{1 \mathrm{c}}$ of $-0.7 \% \pm$ $0.7 \%$ and $-0.5 \% \pm 0.6 \%$, in adolescents and adults, respectively. Adolescent subjects with run-in $\mathrm{HbA}_{1 \mathrm{c}}$ levels of $<7.0 \%, 7.0 \%-7.5 \%$, and $>7.5 \%$ experienced mean changes in $\mathrm{HbA}_{1 \mathrm{c}}$ of $-0.04 \%, 0 \%$, and $-0.98 \%$, respectively. Adult subjects with run-in $\mathrm{HbA}_{1 \mathrm{c}}$ levels of $<7.0 \%, 7.0 \%-7.5 \%$, and $>7.5 \%$ experienced mean changes in $\mathrm{HbA}_{1 \mathrm{c}}$ of $-0.06 \%$, $-0.39 \%$, and $-1.04 \%$, respectively. The percentage of adolescent subjects with $\mathrm{HbA}_{1 \mathrm{c}}$ levels $<7.0 \%$ increased from $16.7 \%$ to $43.3 \%$, and the percentage with levels $>7.5 \%$ decreased from $66.7 \%$ to $20.0 \%$. The percentage of adult subjects with $\mathrm{HbA}_{1 \mathrm{c}}$ levels $<7.0 \%$ increased from $38.3 \%$ to $59.1 \%$, and the percentage with levels $>7.5 \%$ fell from $34.0 \%$ to $10.8 \%$.

Table 2 shows increased TDD from the run-in phase to the study phase for adolescents (from $55.6 \pm 17.1$ to $60.2 \pm 19.8 \mathrm{U}$, $P=0.00146$ ) and adults (from $44.9 \pm 23.7$ to $47.9 \pm 28.0 \mathrm{U}$, $P<0.001$, Wilcoxon signed-rank test). The amount of basal insulin delivered as a percent of TDD, from the run-in phase to the study phase, was reduced in both adolescents (from $49.7 \% \pm 12.1 \%$ to $46.4 \% \pm 8.5 \%, P=0.02271$ ) and adults (from $54.1 \% \pm 10.9 \%$ to $46.8 \% \pm 9.4 \%, P<0.001)$. Mean overall body weight of adolescents increased from $67.4 \pm 13.0$ to $68.4 \pm$ $12.5 \mathrm{~kg}(P=0.06516)$ and that of adults increased from $79.9 \pm$ 18.2 to $81.3 \pm 16.0 \mathrm{~kg}(P<0.001$, Wilcoxon signed-rank test $)$.

\section{Nighttime use of the HCL system}

Table 3 shows the run-in phase and study phase mean \pm SD and median (IQR) SG values, percent of SG values in various ranges, and the within-day SD and CV of SG levels during the night (i.e., period between 10:00 p.m. and 7:00 a.m.). The mean percent of in-target SG values, for adolescents, increased from $64.2 \% \pm 14.1 \%$ to $71.5 \% \pm 10.3 \%(P<0.001)$. A decreased percent of nighttime SG values $>180 \mathrm{mg} / \mathrm{dL}$ (from $30.0 \% \pm 15.6 \%$ to $25.6 \% \pm 9.8 \%, P=0.03199$ ), $\leq 70$ $\mathrm{mg} / \mathrm{dL}$ (from $5.8 \% \pm 5.3 \%$ to $2.9 \% \pm 1.6 \%, P=0.00210$, Wilcoxon signed-rank test), and $\leq 50 \mathrm{mg} / \mathrm{dL}$ (from $1.0 \% \pm$ $1.3 \%$ to $0.6 \% \pm 0.6 \%, P=0.11219$, Wilcoxon signed-rank test) was also observed. For adults, the percent of nighttime in-target SG values increased from $67.6 \% \pm 13.9 \%$ to $76.5 \% \pm$ $9.3 \%(P<0.001)$, while a reduction in values $>180 \mathrm{mg} / \mathrm{dL}$ (from $25.8 \% \pm 15.1 \%$ to $20.4 \% \pm 9.6 \%, P<0.001$, Wilcoxon signed-rank test), $\leq 70 \mathrm{mg} / \mathrm{dL}$ (from $6.6 \% \pm 5.3 \%$ to $3.2 \% \pm$ $2.4 \%, P<0.001$, Wilcoxon signed-rank test), and $\leq 50 \mathrm{mg} / \mathrm{dL}$ (from $1.1 \% \pm 1.5 \%$ to $0.7 \% \pm 0.8 \%, P<0.001$, Wilcoxon signed-rank test) was observed, from the run-in phase to the study phase. These findings indicate reduced exposure to both nighttime hyperglycemia and hypoglycemia during the study phase, when compared to the run-in phase, for both cohorts. Adult nighttime SD $(P=0.00471$, Wilcoxon signed-rank test $)$ and $\mathrm{CV}(P<0.001)$ of SG levels during the study phase were reduced. Adolescent $\mathrm{CV}$ of $\mathrm{SG}$ levels during the night was reduced $(P=0.03646)$.

\section{Fasting $S G$}

The ability of the HCL system to attenuate hyperglycemic exposure was also evidenced by its effect on in-target fasting SG values obtained between 5:00 a.m. and 9:00 a.m. that were not preceded by a bolus of insulin. For adolescents, the proportion of mean fasting in-target $(71-180 \mathrm{mg} / \mathrm{dL}) \mathrm{SG}$ 


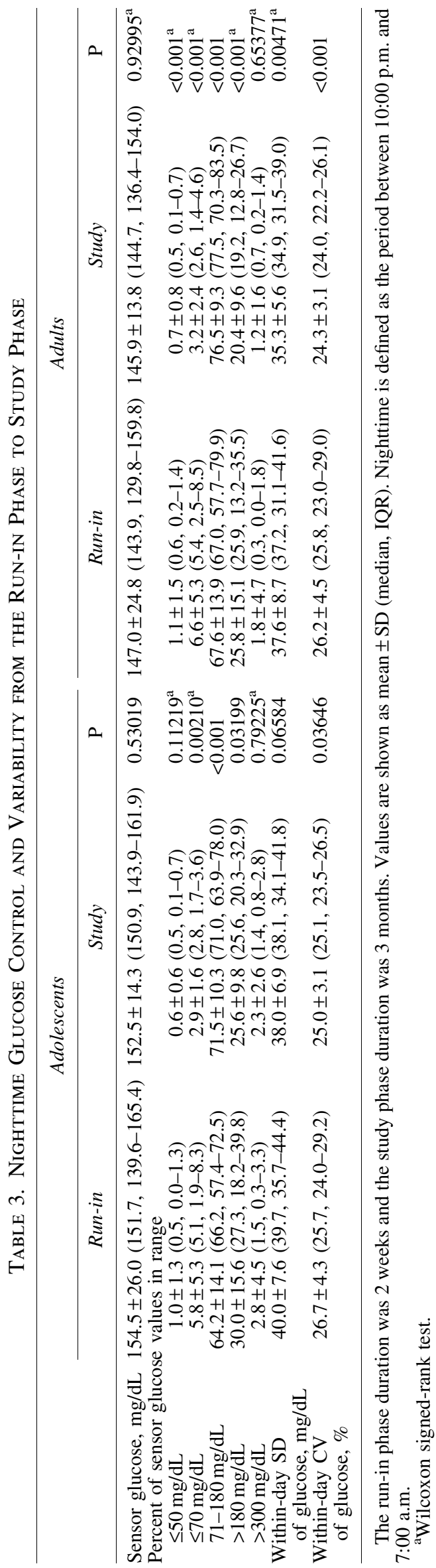

values was $67.9 \% \pm 20.7 \%$ during the run-in phase and $78.8 \% \pm 17.2 \%$ during the study phase, an increase of $12.5 \% \pm$ $14.9 \%(P<0.001)$. The proportion of fasting in-target $S G$ values also increased in adults; from $70.0 \% \pm 21.2 \%$ to $84.4 \% \pm 12.1 \%$, a difference of $14.4 \% \pm 17.8 \% \quad(P<0.001$, Wilcoxon signed-rank test).

\section{i-STAT, SG, and sensor accuracy during FST}

For adolescents, the proportion of in-target glucose values during the hotel stay was $67.4 \% \pm 27.7 \%$ when assessed by iSTAT venous samples and $72.0 \% \pm 11.6 \%$ for SG measurements. The proportion of adolescent in-target SG values during the entire in-home study was $67.2 \% \pm 8.2 \%$. For adults, the proportion of in-target glucose values during the hotel stay was $74.2 \% \pm 17.5 \%$ for i-STAT venous samples and $76.9 \% \pm 8.3 \%$ for SG measurements, both of which were relatively comparable to the proportion of adult in-target SG values during the entire in-home study $(73.8 \% \pm 8.4 \%)$. The lower percent of in-target values observed in adolescents relative to adults, during the hotel FST period, is not unexpected and likely due to higher activity levels and eating.

Overall sensor accuracy, as measured by the MARD \pm SD between SG and i-STAT venous blood glucose values, during the hotel FST, was $11.2 \% \pm 9.7 \%$ (902 paired points) for adolescents and $10.0 \% \pm 8.7 \%$ (2808 paired points) for adults (Table 4). Sensor accuracy within the target range (71$180 \mathrm{mg} / \mathrm{dL}$ ) was $10.5 \% \pm 9.7 \%$ (645 paired points) for adolescents and $9.6 \% \pm 8.3 \%$ (2118 paired points) for adults. Sensor accuracy $>180 \mathrm{mg} / \mathrm{dL}$ was $12.8 \% \pm 9.1 \%$ (245 paired points) for adolescents and $10.3 \% \pm 7.8 \%$ (625 paired points) for adults. Sensor accuracy $\leq 70 \mathrm{mg} / \mathrm{dL}$, as determined by mean absolute difference, was $12.3 \pm 9.2 \mathrm{mg} / \mathrm{dL}$ (12 paired points) for adolescents, and $12.2 \pm 10.6 \mathrm{mg} / \mathrm{dL}$ (65 paired points) for adults.

Overall grouped-cohort sensor accuracy was $10.3 \% \pm$ $9.0 \%$ (3710 paired points) and was similar for data collected during the predefined daytime and nighttime hours, where overall daytime (from 7:00 a.m. to 10:00 p.m.) MARD was $10.4 \% \pm 9.7 \%$ (1787 paired values) and nighttime (i.e., period between 10:00 p.m. and 7:00 a.m.) MARD was 10.2\% $\pm 8.3 \%$ (1923 paired values).

\section{Discussion}

As previously reported, ${ }^{25}$ this pivotal study showed that the HCL system could be used safely by adolescents and adults with type 1 diabetes in an outpatient setting, with no episodes of severe hypoglycemia or diabetic ketoacidosis (DKA) in over 12,000 patient-days of use. In addition, these analyses showed that use of the HCL system increased the percent of in-target SG levels and was associated with clinically important reductions in $\mathrm{HbA}_{1 \mathrm{c}}$ values in both cohorts. These findings and the ability of the system to increase the time in-target range, while reducing the time below and above target levels, support its function as a hyperglycemia and hypoglycemia attenuator. Moreover, HCL control was, for the most part, as effective in adolescents as it was in adults, despite the fact that most adolescents who enrolled in the study had baseline $\mathrm{HbA}_{1 \mathrm{c}}$ levels in excess of the target value $(7.5 \%)$ for their age. Improved $\mathrm{HbA}_{1 \mathrm{c}}$ with an $\mathrm{HCL}$ system was also reported by Thabit et al., ${ }^{18}$ who performed a 
Table 4. Distribution of i-Stat and Sensor Glucose Values, During Hotel Frequent Sample Testing

\begin{tabular}{|c|c|c|c|c|c|c|c|c|}
\hline \multirow[b]{3}{*}{$\begin{array}{l}\text { Reference glucose } \\
\text { range, } \mathrm{mg} / \mathrm{dL}\end{array}$} & \multicolumn{8}{|c|}{ Percent of values in range } \\
\hline & \multicolumn{4}{|c|}{ Adolescents } & \multicolumn{4}{|c|}{ Adults } \\
\hline & $\mathrm{N}$ & $\begin{array}{c}i-S T A T \\
(\text { mean } \pm S D)\end{array}$ & $\mathrm{N}$ & $\begin{array}{c}\text { Sensor } \\
(\text { mean } \pm S D)\end{array}$ & $\mathrm{N}$ & $\begin{array}{c}i-S T A T \\
(m e a n \pm S D)\end{array}$ & $\mathrm{N}$ & $\begin{array}{c}\text { Sensor } \\
(\text { mean } \pm S D)\end{array}$ \\
\hline$\leq 70$ & 12 & $1.2 \pm 2.2$ & 887 & $1.9 \pm 1.5$ & 69 & $2.8 \pm 5.4$ & 4103 & $2.7 \pm 2.1$ \\
\hline $71-180$ & 653 & $67.4 \pm 27.7$ & 33862 & $72.0 \pm 11.6$ & 2147 & $74.2 \pm 17.5$ & 117585 & $76.9 \pm 8.3$ \\
\hline$>180$ & 262 & $31.4 \pm 28.4$ & 12086 & $26.1 \pm 12.1$ & 638 & $23.0 \pm 18.0$ & 31187 & $20.4 \pm 8.5$ \\
\hline
\end{tabular}

Shown are the averages of individual-subject percentage points for the i-STAT blood glucose reference and sensor glucose values, across reference glucose ranges, during the hotel stay for adolescents and adults. One 24-h frequent sample testing occurred during the hotel stay.

$N$, number of i-STAT or sensor glucose values.

study of long enough duration to obtain $\mathrm{HbA}_{1 \mathrm{c}}$ levels, and by Kovatchev et al. ${ }^{14}$ who performed a 6-month closed-loop control study in a limited number of individuals with a low baseline $\mathrm{HbA}_{1 \mathrm{c}}$ of $7.2 \%$ that was $7.0 \%$ at study end. As reported in two other closed-loop studies, ${ }^{6,17}$ we also showed that HCL control reduced glycemic variability, which may have important implications for oxidative stress ${ }^{27}$ and longterm diabetes-related complications. ${ }^{28}$ Based on the data presented, the HCL system could be a transformative therapeutic option for insulin-treated patients.

Ongoing research has allowed the rapid development of automated insulin delivery systems with user interface, safety, and performance attributes that make testing in unmonitored settings possible. As reported in prior studies, the HCL system was capable of safely delivering insulin despite individualized, adapted constraints on maximal insulin delivery, disrupted sensor/pump signal transmission, ${ }^{20}$ exercise challenges, ${ }^{20,29}$ or overcalibration of sensors. ${ }^{29}$ In addition, the performance of the Guardian Sensor 3, as demonstrated during a pivotal trial of sensor performance ${ }^{30}$ and the hotel segment of this study, was improved from earlier generations $^{31,32}$ of glucose sensors. These attributes allowed for the positive safety profile of this pivotal trial.

It is not surprising that the HCL system was effective in lowering the risk of biochemical hypoglycemia during the night in both adolescents and adults, since similar results have been reported with integrated sensor-augmented pump systems that can suspend insulin delivery either at a preset SG threshold $^{33}$ or 30 min in advance of reaching a threshold ${ }^{34}$ to mitigate hypoglycemia. However, in the ASPIRE In-Home study, ${ }^{33}$ and one user evaluation study of the MiniMed 640G pump, ${ }^{34}$ threshold suspend features reduced hypoglycemia, particularly at night, but did not reduce hyperglycemia or, when measured, $\mathrm{HbA}_{1 \mathrm{c}}$ levels. While data on the treatment of hypoglycemic events were not captured, overall, our study showed that the HCL system that automatically increases, decreases, and suspends insulin delivery rates can effectively reduce hypoglycemic and hyperglycemic excursions during the day and night. In-home use of the system also resulted in a reduction in $\mathrm{HbA}_{1 \mathrm{c}}$, and without any episodes of severe hypoglycemia or DKA.

There are several previous studies that have evaluated up to four different HCL or closed-loop systems (including two with both insulin and glucagon) ${ }^{14,17,18,35-38}$; all with small sample sizes between 12 and 52 subjects and only 4 with study durations greater than 2 weeks. Even fewer studies have evalu- ated the effects of systems used continuously (i.e., for the full 24-h day and night periods) across multiple weeks (i.e., 3-24 weeks) in the home setting. ${ }^{14,18,38}$ Comparing our results to previous short-term studies is difficult. Nevertheless, our previous report ${ }^{25}$ and findings herein with a large number of HCL therapy patient-days support the translation of automated insulin delivery technology into commercial products that improve diabetes patient outcomes. The robust data from this pivotal trial of HCL therapy led to the recent FDA approval of the MiniMed 670 G system ${ }^{39}$ in the United States.

Our study's strengths include its multicenter design, the large number of subjects and unmonitored, unrestricted subject-days, the inclusion of adolescents and adults, and the inclusion of a hotel stay in which sensor-derived time-intarget results were confirmed by reference blood glucose measurements. We showed improvements in glycemic control with reductions in hypoglycemia even in a cohort with relatively low initial mean $\mathrm{HbA}_{1 \mathrm{c}}$ levels. We studied a commercial-ready system that integrates a control algorithm within the pump. At the request of enrolled subjects and with the approval of the FDA, this study was modified to include an optional continued access program. Eighty percent of the subjects requested to continue with the HCL system (formal data were not collected as to why subjects did not enter the continued access program), and 96 have been using the system for 1 year or longer.

Limitations to this study included its single-arm, nonrandomized design and the 2-week duration of the run-in phase that was shorter than the 3-month study phase, leading to an imbalance in data quantity. While the absence of severe hypoglycemia in the present study ( 0 events in 12,389 patient-days) compares favorably to recently published observations in patients established on pump therapy with or without use of continuous glucose monitoring, interpretation of this finding is limited by the relatively short duration of the study. Nevertheless, the current study is the longest outpatient 24-h HCL study in more than 100 adolescents and adults. The mean baseline SG $(150.2 \mathrm{mg} / \mathrm{dL})$ may have been better than expected from the mean baseline $\mathrm{HbA}_{1 \mathrm{c}}$ level $(7.4 \%)$, due to patient engagement with the study team during the run-in phase and the fact that a new pump and sensor were being used. If subjects immediately improved glucose control during the run-in phase, due to the use of sensor-augmented pump therapy, it would have underestimated the difference between the Manual Mode- and Auto Mode-enabled periods. While efforts were made to include subjects across different 
HbA1c values, the generalizability of our results may be limited, given the lower baseline HbA1c levels for both cohorts relative to T1D exchange mean HbA1c levels ${ }^{1}$; the frequent contact that study subjects had with site personnel; the fact that approximately half of the adolescents and twothirds of the adults were using CGM at baseline; and the exclusion of subjects with $\mathrm{HbA}_{1 \mathrm{c}}$ levels $>10 \%, \geq 2$ recent episodes of severe hypoglycemia, and those with any recent episodes of diabetic ketoacidosis.

Results of this pivotal trial support and extend those from earlier studies of HCL systems (from Medtronic and other investigators) that were conducted under closely supervised conditions, or for shorter durations, and enrolled fewer subjects. Data reported here suggest that the present system is effective for adolescents and adults and that it may benefit patients as they strive to improve overall glycemic control, while minimizing hypoglycemia. The ability of this integrated system to automatically and safely increase, decrease, and suspend insulin delivery represents an important advance in type 1 diabetes therapy for individuals with diabetes, their families, and their healthcare teams.

\section{Acknowledgments}

The authors thank the study participants, who were willing to help determine the safety and effectiveness of this system in their homes, and acknowledge valuable assistance from staff members at each of the investigational centers. They appreciate the efforts of Medtronic employees: Suiying Huang, Yongyin Wang, Xiaoxiao Chen, Margaret Liu, and Vivian Chen for data management and analysis; Rebecca Gottlieb, PhD, for descriptions of the closed-loop algorithm; and Thomas Troub and Cathy Rogert for assistance in monitoring study conduct.

\section{Author Disclosure Statement}

The following authors: Drs. Garg, Weinzimer, Buckingham, Bode, Bailey, Brazg, Ilany, Slover, Anderson, and Bergenstal served as principal investigators for this study and received compensation and research support from Medtronic. Dr. Tamborlane served as a consultant in the study and received compensation from Medtronic. Drs. Grosman, Roy, Cordero, Shin, Lee, and Kaufman, employees of Medtronic, participated in data analysis and/or critical review of the manuscript.

\section{References}

1. Miller KM, Foster NC, Beck RW, et al.: Current state of type 1 diabetes treatment in the U.S.: Updated data from the T1D Exchange clinic registry. Diabetes Care 2015;38:971-978.

2. Weinzimer SA, Steil GM, Swan KL, et al.: Fully automated closed-loop insulin delivery versus semiautomated hybrid control in pediatric patients with type 1 diabetes using an artificial pancreas. Diabetes Care 2008;31:934-939.

3. Trevitt S, Simpson S, Wood A: Artificial pancreas device systems for the closed-loop control of type 1 diabetes: what systems are in development? J Diabetes Sci Technol 2016; 10:714-723.

4. Kovatchev BP, Breton M, Man CD, et al.: In silico preclinical trials: a proof of concept in closed-loop control of type 1 diabetes. J Diabetes Sci Technol 2009;3:44-55.
5. Atlas E, Nimri R, Miller S, et al.: MD-logic artificial pancreas system: a pilot study in adults with type 1 diabetes. Diabetes Care 2010;33:1072-1076.

6. Breton M, Farret A, Bruttomesso D, et al.: Fully integrated artificial pancreas in type 1 diabetes: modular closed-loop glucose control maintains near normoglycemia. Diabetes 2012;61:2230-2237.

7. Buckingham BA, Beck RW, Ruedy KJ, et al.: Diabetes Research in Children Network Study Group; Type 1 Diabetes TrialNet Study Group. The effects of inpatient hybrid closed-loop therapy initiated within 1 week of type 1 diabetes diagnosis. Diabetes Technol Ther 2013;15: 401-408.

8. Castle JR, Engle JM, El Youssef J, et al.: Novel use of glucagon in a closed-loop system for prevention of hypoglycemia in type 1 diabetes. Diabetes Care 2010;33:12821287.

9. O'Grady MJ, Retterath AJ, Keenan DB, et al.: The use of an automated, portable glucose control system for overnight glucose control in adolescents and young adults with type 1 diabetes. Diabetes Care 2012;35:2182-2187.

10. Russell SJ, El-Khatib FH, Nathan DM, et al.: Blood glucose control in type 1 diabetes with a bihormonal bionic endocrine pancreas. Diabetes Care 2012;35:2148-2155.

11. Kovatchev BP, Renard E, Cobelli C, et al.: Feasibility of outpatient fully integrated closed-loop control: first studies of wearable artificial pancreas. Diabetes Care 2013;36: 1851-1858.

12. Nimri R, Muller I, Atlas E, et al.: Night glucose control with MD-Logic artificial pancreas in home setting: a single blind, randomized crossover trial-interim analysis. Pediatr Diabetes 2014;15:91-99.

13. Phillip M, Battelino T, Atlas E, et al.: Nocturnal glucose control with an artificial pancreas at a diabetes camp. $\mathrm{N}$ Engl J Med 2013;368:824-833.

14. Kovatchev B, Cheng P, Anderson SM, et al.: Feasibility of long-term closed-loop control: a multicenter 6-month trial of 24/7 automated insulin delivery. Diabetes Technol Ther 2016 [DOI: 10.1089/dia.2016.0333].

15. Nimri R, Muller I, Atlas E, et al.: MD-Logic overnight control for 6 weeks of home use in patients with type 1 diabetes: randomized crossover trial. Diabetes Care 2014; 37:3025-3032.

16. Tauschmann M, Allen JM, Wilinska ME, et al.: Day-andnight hybrid closed-loop insulin delivery in adolescents with type 1 diabetes: a free-living, randomized clinical trial. Diabetes Care 2016;39:1168-1174.

17. Thabit H, Elleri D, Leelarathna L, et al.: Unsupervised home use of an overnight closed-loop system over 3-4 weeks: a pooled analysis of randomized controlled studies in adults and adolescents with type 1 diabetes. Diabetes Obes Metab 2015; 17:452-458.

18. Thabit H, Tauschmann M, Allen JM, et al.: Home use of an artificial beta cell in type 1 diabetes. N Engl J Med 2015; 373:2129-2140.

19. Steil GM, Palerm CC, Kurtz N, et al.: The effect of insulin feedback on closed loop glucose control. J Clin Endocrinol Metab 2011;96:1402-1408.

20. Roy A, Grosman B, Parikh N, et al.: Physiological and technical stress test for Medtronic's artificial pancreas system in a supervised outpatient setting. Diabetes Technol Ther 2015; 17:A17.

21. Ly TT, Roy A, Grosman B, et al.: Day and night closedloop control using the integrated Medtronic hybrid closed- 
loop system in type 1 diabetes at diabetes camp. Diabetes Care 2015;38:1205-1211.

22. Ly TT, Weinzimer SA, Maahs DM, et al.: Automated hybrid closed-loop control with a proportional-integral-derivative based system in adolescents and adults with type 1 diabetes: individualizing settings for optimal performance. Pediatr Diabetes 2016;10.1111/pedi.12399.

23. Grosman B, Ilany J, Roy A, et al.: Hybrid closed-loop insulin delivery in type 1 diabetes during supervised outpatient conditions. J Diabetes Sci Technol 2016;10:708-713.

24. de Bock MI, Roy A, Cooper MN, et al.: Feasibility of outpatient 24-hour closed-loop insulin delivery. Diabetes Care 2015;38:e186-e187.

25. Bergenstal RM, Garg S, Weinzimer SA, et al.: Safety of a hybrid closed-loop insulin delivery system in patients with type 1 diabetes. JAMA 2016;316:1407-1408.

26. Ruiz JL, Sherr JL, Cengiz E, et al.: Effect of insulin feedback on closed-loop glucose control: a crossover study. J Diabetes Sci Technol 2012;6:1123-1130.

27. Dasari PS, Gandomani BS, Teague AM, et al.: Glycemic variability is associated with markers of vascular stress in adolescents. J Pediatr 2016;172:47-55.

28. Jung HS: Clinical implications of glucose variability: chronic complications of diabetes. Endocrinol Metab 2015; 30:167-174.

29. de Bock M, Dart J, Roy A, et al.: Exploration of the performance of a hybrid closed loop insulin delivery algorithm that includes insulin delivery limits designed to protect against hypoglycemia. J Diabetes Sci Technol 2016 [DOI: 10.1177/1932296816668876].

30. Brazg R, Christiansen M, Bode B, et al.: Accuracy of a fourth-generation glucose sensor throughout its functional life. Diabetes 2016;65:A226.

31. Bailey TS, Ahmann A, Brazg R, et al.: Accuracy and acceptability of the 6-day Enlite continuous subcutaneous glucose sensor. Diabetes Technol Ther 2014;16:277-283.

32. Keenan DB, Mastrototaro JJ, Zisser H, et al.: Accuracy of the enlite 6-day glucose sensor with Guardian and Veo calibration algorithms. Diabetes Technol Ther 2012;14: 225-231.

33. Bergenstal RM, Klonoff DC, Garg SK, et al.: Thresholdbased insulin-pump interruption for reduction of hypoglycemia. N Engl J Med 2013;369:224-232.

34. Choudhary P, Olsen BS, Conget I, et al.: Hypoglycemia prevention and user acceptance of an insulin pump system with predictive low glucose management. Diabetes Technol Ther 2016;18:288-291.

35. Kovatchev BP, Renard E, Cobelli C, et al.: Safety of outpatient closed-loop control: first randomized crossover trials of a wearable artificial pancreas. Diabetes Care 2014;37: 1789-1796.

36. Russell SJ, El-Khatib FH, Sinha M, et al.: Outpatient glycemic control with a bionic pancreas in type 1 diabetes. $\mathrm{N}$ Engl J Med 2014;371:313-325.

37. van Bon AC, Luijf YM, Koebrugge R, et al.: Feasibility of a portable bihormonal closed-loop system to control glucose excursions at home under free-living conditions for 48 hours. Diabetes Technol Ther 2014;16:131-136.

38. Tauschmann M, Allen JM, Wilinska ME, et al.: Home use of day-and-night hybrid closed-loop insulin delivery in suboptimally controlled adolescents with type 1 diabetes: a 3-week, free-living, randomized crossover trial. Diabetes Care 2016;39:2019-2025.

39. Food and Drug Administration, Center for Devices and Radiological Health: MiniMed 670G System approval letter, September 28, 2016. www.accessdata.fda.gov/cdrh_docs/ pdf16/P160017a.pdf (accessed January 11, 2017).

Address correspondence to: Satish K. Garg, MD Barbara Davis Center for Diabetes University of Colorado Denver 1775 Aurora Court Aurora, CO 80045

E-mail: satish.garg@ucdenver.edu 